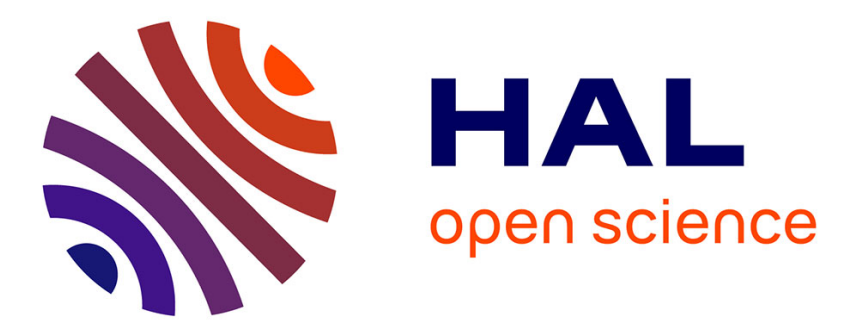

\title{
Modelling of the heat build-up temperature and damage fields in bulk filled-rubber samples during fatigue
}

\author{
C. Ovalle Ovalle Rodas, Q. Guo, F. Zaïri, X. Guo, P. Charrier
}

\section{To cite this version:}

C. Ovalle Ovalle Rodas, Q. Guo, F. Zaïri, X. Guo, P. Charrier. Modelling of the heat build-up temperature and damage fields in bulk filled-rubber samples during fatigue. European Conference on Constitutive Models for Rubbers, Jun 2019, Nantes, France. pp.379-384, 10.1201/9780429324710-66 . hal-02507319

HAL Id: hal-02507319

https://hal-mines-paristech.archives-ouvertes.fr/hal-02507319

Submitted on 13 Mar 2020

HAL is a multi-disciplinary open access archive for the deposit and dissemination of scientific research documents, whether they are published or not. The documents may come from teaching and research institutions in France or abroad, or from public or private research centers.
L'archive ouverte pluridisciplinaire HAL, est destinée au dépôt et à la diffusion de documents scientifiques de niveau recherche, publiés ou non, émanant des établissements d'enseignement et de recherche français ou étrangers, des laboratoires publics ou privés. 


\title{
Modelling of the heat build-up temperature and damage fields in bulk filled-rubber samples during fatigue
}

\author{
C. Ovalle Rodas \\ PSL Research University, Mines ParisTech, Centre des Matériaux \\ CNRS 7633 BP 87, 91003 Evry, France \\ Q. Guo \& F. Zaïri \\ Lille University, Civil Engineering and geo-Environmental Laboratory (EA 4515 LGCgE), 59000 Lille, France
}

X. Guo

Dalian University of Technology, Department of Engineering Mechanics, 116024 Dalian, China

P. Charrier

Groupe Vibracoustic, Société Vibracoustic Nantes, Service CAE Durability Prediction

Zone Industrielle Nantes Carquefou BP 419, 44474 Carquefou, France

\begin{abstract}
In the present work, a finite strain thermo-viscoelastic-damage model is used to predict the cyclic thermo-mechanical response of bulk rubber samples during fatigue. The model parameters were identified and verified using experimental observations on stress-softening, hysteresis and dissipative heating obtained in thin rubber samples containing different amounts of carbon-black and cyclically loaded under different minimum stretch levels. Predicted evolutions of the heat build-up temperature and damage fields in bulk rubber samples during fatigue are discussed.
\end{abstract}

\section{INTRODUCTION}

Rubber materials are commonly submitted to cyclic loading in the automotive and aeronautical industries. Depending on the boundary conditions, heat buildup is induced under cyclic loading as a consequence of the thermo-mechanical coupling and the viscous properties of the material (Ovalle Rodas et al. 2013). The physical consequences of heat build-up include the degradation of the rubber mechanical properties.

In previous contributions (Ovalle Rodas et al. 2014, Ovalle Rodas et al. 2016), a thermodynamically consistent finite strain thermo-viscoelastic model was developed to predict the heat build-up field in thin rubber samples during fatigue. The proposed model was then enhanced to account for the damage effect on the cyclic stress-softening (Guo, Zaïri, \& Guo 2018). Using a viscous dilatation tensor, related to the timedependent response of the rubber material, and a fatigue damage indicator, related to the internal network damage kinetics, both the thermo-mechanical response and the damage-related stress-softening was predicted for different stretch levels. The viscoelastic and damage dissipations contributing to heat build- up were considered in the model formulation using the internal state variable theory. The general form of the model was represented by a thermal branch responsible for the stress-free thermal expansion joined to parallel mechanical multi-branches responsible for the cyclically damaged-elastic response and the inelastic effects, i.e. fatigue-induced stresssoftening and hysteresis. An interpretation of the underlying physical mechanisms was proposed in which two types of dissipative network rearrangements are considered, i.e. recoverable rearrangements inducing viscoelasticity and unrecoverable rearrangements inducing damage. In the present work, a finite strain thermo-viscoelastic-damage model is used to predict the cyclic thermo-mechanical response of bulk rubber samples during fatigue. The model parameters were identified and verified using experimental data on stress-softening, hysteresis and dissipative heating obtained in thin rubber samples containing different amounts of carbon-black and cyclically loaded under different stretch levels. Predicted evolutions of the heat build-up temperature and damage fields in bulk rubber samples during fatigue are discussed. 


\section{CONSTITUTIVE MODEL}

The thermo-mechanical response is described by connecting in series a thermal resistance to a generalized Zener-type rheological model, see Fig. 1. The thermal resistance is related to the stress-free thermal expansion and the Zener-type rheological model is related to the isothermal viscoelastic-damage effects.

\section{$2.1 \quad$ Kinematics}

Given the nonlinear deformation map $\mathbf{x}=\varphi(\mathbf{X}, t)$ which maps a material point from its initial position $\mathbf{X}$ in the reference configuration, at a uniform absolute temperature $T_{0}$ taken as a reference, to its actual position $\mathbf{x}$ in the current configuration. The mapping of a material point is given by the deformation gradient $\mathbf{F}=\nabla_{X} \varphi(\mathbf{X}, t)$ and the thermo-mechanical coupling can be considered by using the deformation gradient separation concept (Lee \& Liu 1967):

$\mathbf{F}=\mathbf{F}_{M} \mathbf{F}_{T}$

where

$\mathbf{F}_{T}=F_{T} \mathbf{I}, F_{T}=\exp \left(\int_{T_{0}}^{T} \alpha(u) d u\right)$

is the stress-free thermal expansion part, in which $\mathbf{I}$ is the identity tensor, $\alpha$ is the temperature-dependent coefficient of the thermal expansion and $T$ is the current absolute temperature; and

$\mathbf{F}_{M}=\mathbf{F}_{e}^{i} \mathbf{F}_{v}^{i}$

is the isothermal stress-induced part of each Maxwell element, in which the subscripts $e$ and $v$ denote respectively the elastic and the viscous deformation gradients. In the following, the superscripts are commonly omitted. Due to mechanical incompressibility, the Jacobian of the mechanical transformation $J_{M}=\operatorname{det} \mathbf{F}_{M}$ is equal to unity and that of the total transformation $J=\operatorname{det} \mathbf{F}$ is equal to $F_{T}$.

The corresponding kinematic rate can be written as:

$$
\begin{aligned}
\mathbf{L} & =\mathbf{L}_{e}+\mathbf{L}_{v}+\mathbf{L}_{T} \\
& =\dot{\mathbf{F}}_{e} \mathbf{F}_{e}^{-1}+\mathbf{F}_{e} \dot{\mathbf{F}}_{v} \mathbf{F}_{v}^{-1} \mathbf{F}_{e}^{-1}+\mathbf{F}_{e} \mathbf{F}_{v} \dot{\mathbf{F}}_{T} \mathbf{F}_{T}^{-1} \mathbf{F}_{v}^{-1} \mathbf{F}_{e}^{-1}
\end{aligned}
$$

in which the dot denotes the time derivative. The viscous velocity gradient $\mathbf{L}_{v}$ of each Maxwell element is expressed as:

$\mathbf{L}_{v}=\mathbf{D}_{v}+\mathbf{W}_{v}$

where $\mathbf{D}_{v}$ (symmetric tensor) and $\mathbf{W}_{v}$ (skew tensor) are respectively the viscous deformation rate and the viscous spin. Assuming the viscous flow in each Maxwell branch irrotational (Gurtin \& Anand 2005), i.e. $\mathbf{W}_{v}=\mathbf{0}$, the viscous deformation gradient $\mathbf{F}_{v}$ of each Maxwell element is then extracted:

$\dot{\mathbf{F}}_{v}=\mathbf{F}_{e}^{-1} \mathbf{D}_{v} \mathbf{F}_{e} \mathbf{F}_{v}$

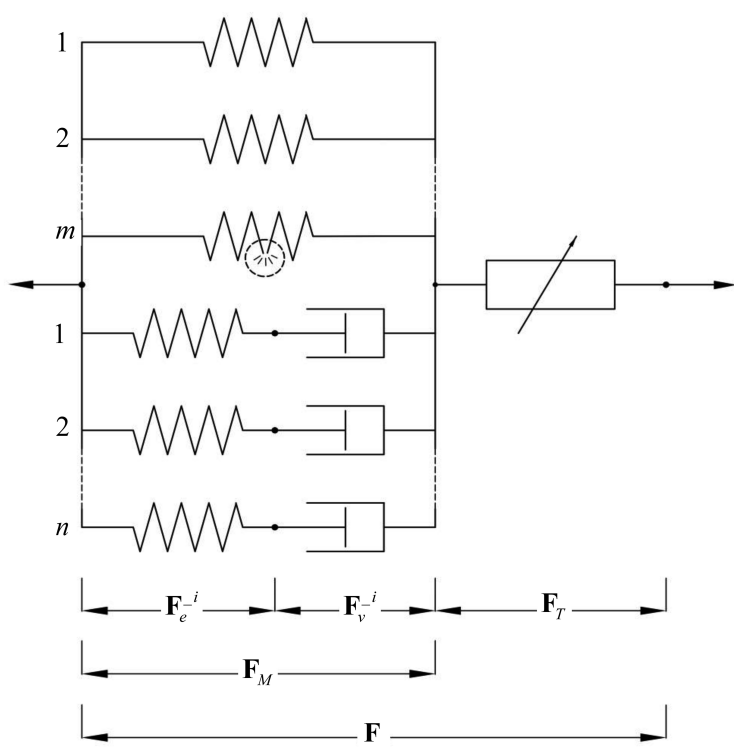

Figure 1: Thermo-viscoelastic-damage model.

\subsection{Model formulation}

To formulate the constitutive model, let us first consider the Helmholtz free energy

$\psi=\psi_{T}(T)+\psi_{M}\left(T, \mathbf{F}_{M}, \mathbf{F}_{v}, \chi\right)$

where the thermal energy function is expressed as:

$\psi_{T}(T)=-\int_{T_{0}}^{T} C_{0}(u)(T-u) \frac{d u}{u}$

in which $C_{0}$ is the specific heat capacity at constant deformation, and the mechanical free energy function $\psi_{M}$ is given by the sum of $m$ relaxed energy functions $\psi_{r}$ and $n$ viscous energy functions $\psi_{v}$ :

$$
\begin{aligned}
\psi_{M}\left(T, \mathbf{F}_{M}, \mathbf{F}_{v}, \chi\right) & =\sum_{j}^{m} \psi_{r}^{j}\left(T, \mathbf{F}_{M}, \chi\right)+ \\
& +\sum_{i}^{n} \psi_{v}^{i}\left(T, \mathbf{F}_{M}, \mathbf{F}_{v}^{i}\right)
\end{aligned}
$$

in which the number of relaxed branches $m$ is a function of the internal state variable $\chi$ to specify the progressive vanishing of relaxed branches due to breakdown of bonds in the interlinked chains.

The relaxed free energy function $\psi_{r}$ is governed by a damage-dependent Arruda \& Boyce (1993) based formulation which considers the resistance to deformation of an eight-chain network:

$\psi_{r}=n_{r} k_{B} T N_{r}\left(\zeta_{r} \frac{\lambda_{r}}{\sqrt{N_{r}}}+\ln \frac{\zeta_{r}}{\sinh \zeta_{r}}\right)$

where $n_{r}=n_{r}(\chi)$ is the number of chains per unit volume (i.e. chain density), $k_{B}$ is the Boltzmann constant, $N_{r}=N_{r}(\chi)$ is the number of segments per chain (i.e. chain length), $\lambda_{r}=\sqrt{\operatorname{trace}\left(\mathbf{F}_{M}^{T} \mathbf{F}_{M}\right) / 3}$ is 
the average stretch on each chain in the relaxed network and $\zeta_{r}=\mathcal{L}^{-1}\left(\lambda_{r} / \sqrt{N_{r}}\right)$ is the inverse Langevin function. The stiffness of the equivalent relaxed network being denoted $C_{r}(\chi)=n_{r}(\chi) k_{B} T$. The evolution in chain density results in an inverse evolution in chain length as a result of the mass conservation law: $N_{r}(\chi) n_{r}(\chi)=n_{r 0} N_{r 0}$, where $n_{r 0}$ and $N_{r 0}$ are reference values. The network damage kinetics is formulated as follows:

$N_{r}(\chi)=N_{r 0}+N_{r \chi}(\chi), N_{r \chi}(\chi)=\chi N_{r 0}$

with $\chi_{0}=\left.\chi\right|_{t=t 0}=0$.

The viscous free energy function $\psi_{v}$ of each Maxwell network takes the following form:

$\psi_{v}=n_{v} k_{B} T N_{v}\left(\zeta_{v} \frac{\lambda_{e}}{\sqrt{N_{v}}}+\ln \frac{\zeta_{v}}{\sinh \zeta_{v}}\right)$

where $n_{v}$ and $N_{v}$ are physical constants of each Maxwell network superimposed on the relaxed network, $\lambda_{e}=\sqrt{\operatorname{trace}\left(\mathbf{F}_{e}^{T} \mathbf{F}_{e}\right) / 3}$ is the elastic stretch and $\zeta_{v}=\mathcal{L}^{-1}\left(\lambda_{e} / \sqrt{N_{v}}\right)$ is the inverse Langevin function. The time-dependent stiffness of each Maxwell network being denoted $C_{v}=n_{v} k_{B} T$.

Thermodynamic formulations for the constitutive equations are based on the Clausius-Duhem inequality. The dissipation $d$ is given, in a mixed description, by:

$d=-\rho(\dot{\psi}+\dot{T} \eta)+\boldsymbol{\pi}: \dot{\mathbf{F}}-\frac{1}{T} \mathbf{q} \cdot \nabla_{X} T \geq 0$

where $\rho$ is the mass density, $\eta$ is the entropy, $\boldsymbol{\pi}$ is the first Piola-Kirchhoff stress, $\mathbf{q}$ is the Piola-Kirchhoff heat flux and $\nabla_{X} T$ is the temperature gradient.

Inserting the expression for the time rate of the specific free energy:

$\dot{\psi}=\frac{\partial \psi}{\partial \mathbf{F}_{M}}: \dot{\mathbf{F}}_{M}+\frac{\partial \psi}{\partial \mathbf{F}_{v}}: \dot{\mathbf{F}}_{v}+\frac{\partial \psi}{\partial \chi} \dot{\chi}+\frac{\partial \psi}{\partial T} \dot{T}$

into (13), and after a series of straightforward derivations it returns:

$$
\begin{aligned}
d & =\left(F_{T} \boldsymbol{\pi}-\rho \frac{\partial \psi}{\partial \mathbf{F}_{M}}\right): \dot{\mathbf{F}}_{M}-\rho\left(\eta+\frac{\partial \psi}{\partial T}-\alpha \varphi\right) \dot{T} \\
& -\sum_{i} \rho \frac{\partial \psi}{\partial \mathbf{F}_{v}^{i}}: \dot{\mathbf{F}}_{v}^{i}-\rho \frac{\partial \psi}{\partial \chi} \dot{\chi}-\frac{1}{T} \mathbf{q} \cdot \nabla_{X} T \geq 0
\end{aligned}
$$

in which $\varphi=1 / \rho \boldsymbol{\pi}: \mathbf{F}$. In order to satisfy this inequality for arbitrary values of the time derivatives of $\mathbf{F}_{M}$ and $T$, the Coleman \& Noll (1963) procedure is used which leads to two potential relations and two dissipation inequalities:

$$
\boldsymbol{\pi}=\frac{\rho}{F_{T}} \frac{\partial \psi}{\partial \mathbf{F}_{M}} \quad \eta=\alpha \varphi-\frac{\partial \psi}{\partial T}
$$

$d_{1}=\sum_{i} \mathbf{A}_{v}^{i}: \dot{\mathbf{F}}_{v}^{i}+\kappa \dot{\chi} \geq 0 \quad d_{2}=-\frac{1}{T} \mathbf{q} \cdot \nabla_{X} T \geq 0$ where $d_{1}$ and $d_{2}$ are referred to as the intrinsic dissipation and the thermal dissipation, respectively, and

$\mathbf{A}_{v}^{i}=-\rho \frac{\partial \psi}{\partial \mathbf{F}_{v}^{i}} \quad \kappa=-\rho \frac{\partial \psi}{\partial \chi}$

denote respectively the thermodynamic conjugate stress-like quantities for $\mathbf{F}_{v}^{i}$ and $\chi$.

These relations form a basis for the development of a suitable theory. They are completed by the evolution law for the viscous deformation rate $\mathbf{D}_{v}$ of each Maxwell network controlling the history-dependent movements of the superimposed free chains and resulting in the time-dependent deviation from the relaxed state:

$\mathbf{D}_{v}=\dot{\gamma}_{v} \mathbf{N}, \quad \mathbf{N}=\frac{\mathbf{T}_{v}^{\prime}}{\sqrt{2} \tau_{v}}, \quad \mathbf{T}_{v}=\frac{\rho}{J} \frac{\partial \psi_{v}}{\partial \mathbf{F}_{M}} \mathbf{F}_{M}$

in which $\mathbf{N}$ is the direction tensor of $\mathbf{D}_{v}$ aligned with the deviatoric part $\mathbf{T}_{v}^{\prime}$ of the viscous Cauchy stress $\mathbf{T}_{v}$ of each Maxwell network, $\tau_{v}=\sqrt{\operatorname{trace}\left(\mathbf{T}_{v}^{\prime} \mathbf{T}_{v}^{\prime}\right) / 2}$ is the effective stress and $\dot{\gamma}_{v}$ is the accumulated viscous strain rate in the Maxwell network which can be formulated using an accumulated viscoplastic strain rate but without yield surface, in which elastic and viscous strain rates are non-zero at all stages of loading. Motivated by reptational dynamics, the stretchdependency of the effective viscosity and the stressdependency of the energy activated process can be both accounted for by using the form of the Bergstrom \& Boyce (1998) power law:

$\dot{\gamma}_{v}=r_{v}\left(\frac{1}{\lambda_{v}-1}\right)^{d_{v}}\left(\tau_{v}\right)^{m_{v}}$

where $r_{v}, d_{v}, m_{v}$ are the viscous material constants of each Maxwell network, and $\lambda_{v}=\sqrt{\operatorname{trace}\left(\mathbf{F}_{v} \mathbf{F}_{v}^{T}\right) / 3}$.

In (16), the intrinsic dissipation $d_{1}$ is the sum of two terms: the first one represents the dissipated mechanical energy due to the viscous-related macro-behaviour and the second one represents the internal work associated with the unrecoverable network rearrangements on the molecular level. Substantively, theses two physical mechanisms are co-existing and complexly coupled to each other. Nevertheless, it is assumed, for the sake of simplicity, that the second term in (16) is proportional to the first one:

$\kappa \dot{\chi}=(\beta-1) \sum_{i} \mathbf{A}_{v}^{i}: \dot{\mathbf{F}}_{v}^{i}$

where $\beta$ is a positive proportional coefficient, similar to the so-called Taylor-Quinney coefficient. As a consequence, the intrinsic dissipation inequality in (16) can be rewritten as

$d_{1}=\beta \sum_{i} \mathbf{A}_{v}^{i}: \dot{\mathbf{F}}_{v}^{i} \geq 0$ 
In addition, due to Taylor assumption inherent to the proposed model, the total Cauchy stress $\mathbf{T}=J^{-1} \boldsymbol{\pi} \mathbf{F}$ in the material is the sum of the Cauchy stresses of all networks:

$$
\mathbf{T}=\mathbf{T}_{r}+\sum_{i} \mathbf{T}_{v}^{i}+p \mathbf{I}
$$

where $p$ is an arbitrary scalar determined from boundary conditions and $\mathbf{T}_{r}$ is the relaxed Cauchy stress:

$\mathbf{T}_{r}=\frac{\rho}{J} \frac{\partial \psi_{r}}{\partial \mathbf{F}_{M}} \mathbf{F}_{M}$

In order to satisfy the first law of thermodynamics, combining $\psi=e-T \eta$, in which $e$ is the internal energy, and (13) an alternative entropy balance equation is obtained:

$\rho \dot{\eta} T=d_{1}-\operatorname{Divq}$

Keeping in mind that all the thermodynamic potentials are functions of the selected state variables, i.e. $T, \mathbf{F}_{M}, \mathbf{F}_{v}$ and $\chi$, and applying repeatedly the chain rule of differentiation, we can deduce a coupled temperature evolution equation from (24):

$$
\begin{aligned}
\rho C \dot{T} & =d_{1}-\operatorname{Divq}+T\left(F_{T} \frac{\partial \boldsymbol{\pi}}{\partial T}-\alpha \rho \frac{\partial \phi}{\partial \mathbf{F}_{M}}\right): \dot{\mathbf{F}}_{M} \\
& -T \sum_{i}\left(\frac{\partial \mathbf{A}_{v}^{i}}{\partial T}+\alpha \rho \frac{\partial \varphi}{\partial \mathbf{F}_{v}^{i}}\right): \dot{\mathbf{F}}_{v}^{i} \\
& -T\left(\frac{\partial \kappa}{\partial T}+\alpha \rho \frac{\partial \varphi}{\partial \chi}\right) \dot{\chi}
\end{aligned}
$$

where $C=T \partial \eta / \partial T$ is a heat capacity-related coefficient, $\phi=\varphi-\psi$ is the complementary energy function with $\varphi=1 / \rho \boldsymbol{\pi}: \mathbf{F}$. In fact, the last three righthand terms of (25) represent, respectively, the thermoelastic heating, the thermoviscoelastic heating, and the heating induced by the coupling of the unrecoverable network rearrangements with the temperature. For the cyclic loading with constant amplitude, all the deformations are changing almost recurrently. Thus, after one complete loading cycle, the changes of the thermodynamics potentials are only induced by the temperature change and the unrecoverable network rearrangements. Since the evolutions of both the temperature and the microstructure after one complete loading cycle are imperceptible, it is reasonable to neglect these three terms:

$\rho C \dot{T}=d_{1}-\operatorname{Divq}$

Finally, the Piola-Kirchhoff heat flux q is given by the Fourier law:

$\mathbf{q}=-\mathbf{K}_{X} \cdot \nabla_{X} T, \quad \mathbf{K}_{X}=\mathbf{F}^{-1} \mathbf{K F}^{-T}$ where $\mathbf{K}_{X}$ is the conductivity tensor in the reference configuration and $\mathbf{K}=k \mathbf{I}$ is the isotropic Euler conductivity tensor, $k$ being the material conductivity factor.

The thermo-mechanical model was implemented into the finite element code MSC.Marc, by means of a strain energy function subroutine in parallel with an internal heat generation subroutine.

\section{EXPERIMENTAL DATA}

\subsection{Identification}

A series of tests have been performed on $2 \mathrm{~mm}$ thickness flat dog-bone shaped specimens to assess the influence of minimum stretch and filler content on the mechanical response evolution, in terms of stress-softening and hysteresis, and on the heat buildup (Guo et al. 2018). The weak thickness avoids high temperature gradients in the transverse direction and allows to extract the intrinsic thermo-mechanical constitutive response. The studied rubber is a carbonfilled SBR reinforced with three amounts of carbonblack: 15, 25 and $43 \mathrm{phr}$ (part per hundred of rubber in weight) corresponding to a carbon-black volume fraction $v_{f}$ of $9.16,14.39$ and $22.42 \%$, respectively. These specimens are referred to as SBRx, where $\mathrm{x}$ is the filler content in phr. Pre-stretched cyclic strain controlled tests have been performed at room temperature to characterize the fatigue response and the surface heat build-up of the carbon-filled SBR along with the minimum stretch dependency. The details concerning the measurements can be found elsewhere (Guo et al. 2018). Some experimental results are used to obtain the needed material constants for the constitutive model while others serve to verify its predictive capabilities (Guo et al. 2018).

In the identification exercise, the network parameters $n_{r}$ and $N_{r}$ were firstly identified using the relaxed-response extracted from the multi-step stress relaxation tests under a loading-unloading stretching cycle. The relaxed stiffness, deduced from the slope of the purely elastic curve of each SBR material, evolves as a power function of the carbon-black volume fraction $v_{f}$. The filler-dependency of the relaxed stiffness may be captured by the following equation:

$C_{r}=n_{r} k_{B} T X\left(v_{f}\right)$

in which $X$ corresponds to the empirical form of the amplification factor initially proposed by Guth (1945) and given by the following general empirical form:

$X\left(v_{f}\right)=1+0.67 c v_{f}+1.62 c^{2} v_{f}^{2}$

in which $c \geq 1$ is a shape factor considering the filler agglomeration.

We assume that only the time-dependent stiffness of the Maxwell networks is affected by the carbonblack content; all other viscous parameters are taken 

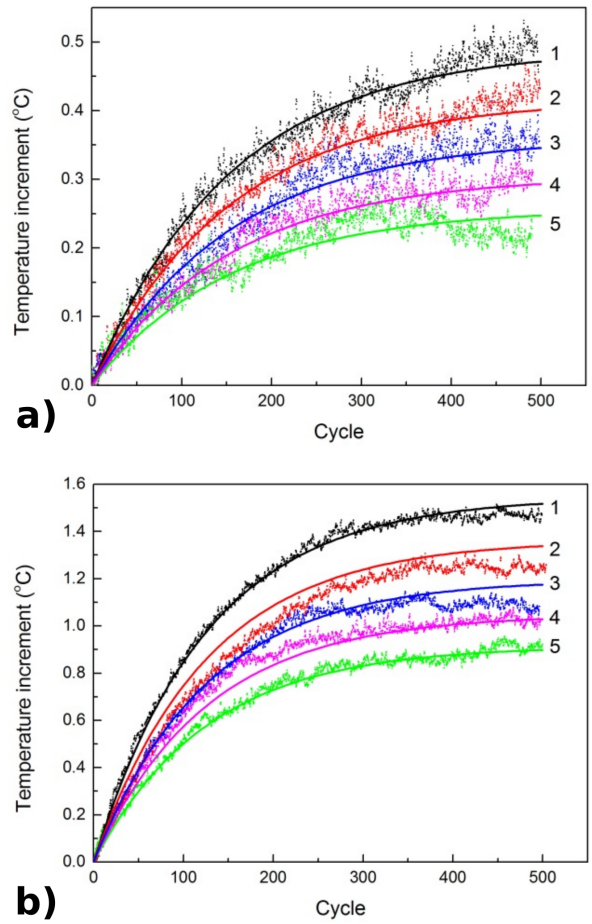

Figure 2: Experimental and simulated surface temperature at different minimum stretches $\left(1: \lambda_{\min }=1.1, \ldots, 5: \lambda_{\min }=1.5\right)$ : (a) SBR15; and (b) SBR43.

filler-independent. The identification of the viscous parameters is performed using the stress-softening and hysteresis data of the $\mathrm{SBR}_{43}$ material. They were successively iterated, by means of trial and error, in order to achieve optimal agreement between the simulations and the experimental curves. Once the $\mathrm{SBR}_{43}$ model parameters are known, the time-dependent stiffness of the Maxwell elements is then predicted using the amplified-inspired approach according to the following equations:

$C_{v}^{1}=n_{v}^{1} k_{B} T X\left(v_{f}\right), \quad C_{v}^{2}=n_{v}^{2} k_{B} T X\left(v_{f}\right)$

in which $X$ is again the empirical amplification factor with $c=2.75$. The remaining model parameters are related to the thermal properties. The thermal expansion coefficient $\alpha$ is extracted from the thermal dilatation response slope in terms of the axial dilatation as a function of the absolute temperature. The remaining thermal values are taken from (Bérardi et al. 1996). The amount of mechanical energy dissipated as heat energy during a cycle is controlled by the coefficient $\beta$ taken equal to 1.003 .

The values of model parameters are reported in Table 1 . The same set is used to capture the inelastic fatigue effects in the three carbon-filled SBR materials.

\subsection{Verification on flat specimens}

In order to demonstrate the capability of the proposed model to predict heat build-up temperature, the experimental and simulated surface temperature at different minimum stretches are shown in Fig. 2. See (Guo et al. 2018), for more details about the ability
Table 1: Mechanical and thermal parameters.

\begin{tabular}{lllll}
\hline Mechanical & & & Thermal \\
\cline { 1 - 2 }$N_{r}=N_{v}^{1}=N_{v}^{2}$ & 6 & & $\alpha\left(\mathrm{K}^{-1}\right)$ & $3.6 \times 10^{-4}$ \\
$n_{r} k_{B} T(\mathrm{MPa})$ & 0.344 & & $k\left(\mathrm{~W} \mathrm{~m}^{-1} \mathrm{~K}^{-1}\right)$ & 0.19 \\
$n_{v}^{1} k T(\mathrm{MPa})$ & 0.105 & & $\rho C\left(\mathrm{~J} \mathrm{~m}^{-3} \mathrm{~K}^{-1}\right)$ & $1.78 \times 10^{6}$ \\
$n_{v}^{2} k T(\mathrm{MPa})$ & 0.231 & & $h\left(\mathrm{~W} \mathrm{~m}^{-2} \mathrm{~K}^{-1}\right)$ & 13 \\
$r_{v}^{1}\left(\mathrm{MPa}^{-2} \mathrm{~s}^{-1}\right)$ & 6 & & \\
$r_{v}^{2}\left(\mathrm{MPa}^{-2} \mathrm{~s}^{-1}\right)$ & 530 & & \\
$d_{v}^{1}=d_{v}^{2}$ & 0.01 & & \\
$m_{v}^{1}=m_{v}^{2}$ & 2 & & \\
\hline
\end{tabular}

of the model to capture, over an entire range of minimum stretch levels, the filler content effect on, respectively, the stress-softening, the hysteresis and the surface heat build-up as a function of the cycle number.

\section{HEAT BUILD-UP AND DAMAGE SIMULATIONS}

The above results show the ability of the model to capture and predict the significant features of the cyclic response and the heat build-up of carbon-filled SBR. The predictive capability of the model over a range of filler concentrations is also demonstrated. Flat specimens involve very weak gradients in the transverse direction; besides, in the case of bulk structures significant gradients may be generated. In order to illustrate this aspect, we propose now to analyze how the triaxial stress state may influence the inelastic fatigue effects in relation with the cyclic deformation mechanisms. Triaxial stress states different from the uniaxial state can be induced by the sample shape: Cylindrical hourglass-shaped samples AE2 and AE42 present two different curvature radii, respectively $R=2 \mathrm{~mm}$ and $R=42 \mathrm{~mm}$, in order to set different triaxial stress states in the median crosssection. The lower the curvature radius, the higher is the hydrostatic stress (high triaxial state) in the specimen. In our simulations, the same equivalent strain in the median cross-section is applied for the two specimens.

Fig. 3 illustrates the damage and temperature evolution predictions at the centre and on the surface of the median cross-section. The cyclic dissipation depends on the stress state induced by the sample shape. A centre-to-surface gradient is therefore expected according to the sample curvature radius, with a dissipation higher on the surface than in the centre. Indeed, the damage distribution exhibits higher damage values on the sample surface whatever the sample shape and with a centre-to-surface gradient higher for the smallest sample curvature radius. Fig. 3a shows a nonlinear evolution of the damage under cyclic loading with a continuous increase of the difference between the centre and the surface. Although the cyclic dissipation transferred into heat energy is larger on the sample surface, the thermal convection at the surface with the environment, in parallel with the low material 

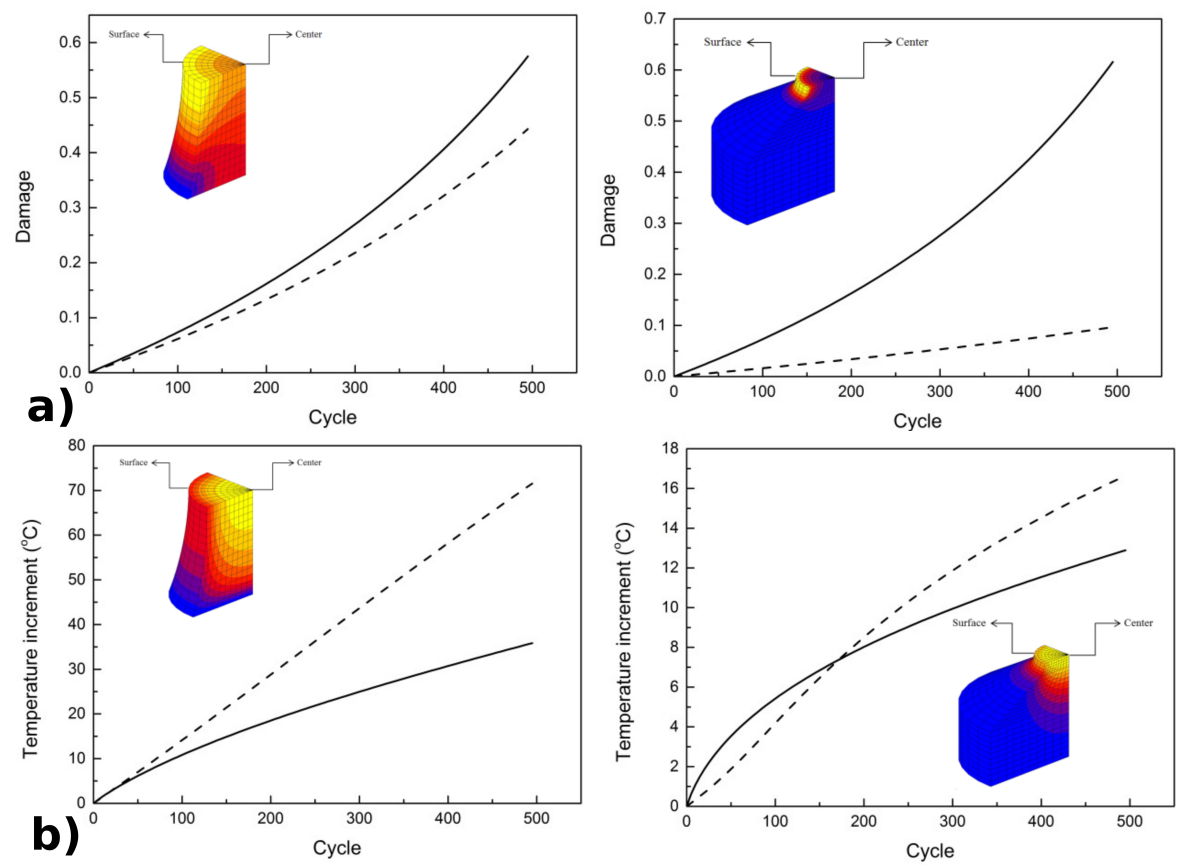

Figure 3: Simulated (a) damage and (b) temperature increment in the AE42 and AE2 specimens (surface: continuous lines, center: dashed lines).

conductivity factor, makes the heat energy concentrates in the core and causes the higher temperature on the sample centre. This thermal insulator feature of the rubber implies significant thermal gradients in the bulkiest sample. Besides, the high temperatures inside the sample could be sufficiently important to induce thermal ageing effects (Neuhaus et al. 2017). As shown in Fig. 3b, the temperature increases nonlinearly under cyclic loading with a decreasing rate larger on the surface than in the centre due to thermal convection. At the beginning of the cyclic loading, large dissipation gradients induce higher surface temperature on the surface than in the centre.

\section{CONCLUSIONS}

A thermo-viscoelastic-damage constitutive model was presented to describe the cyclic dissipation in rubbers in connection to the network rearrangements. The model was identified and verified using experimental data obtained on a carbon-black filled SBR in the form of flat specimens containing different amounts of carbon-black and cyclically loaded under different minimum stretch levels. Numerical applications were carried out on bulk specimens with two different curvature radii in order to set different triaxial stress states in the median cross-section.

The presented model provides a useful tool for damage and thermal patterns estimation in rubber structures. A quantitative evaluation of the model remains however an important issue for future works.

\section{REFERENCES}

Arruda, E. M. \& M. C. Boyce (1993). A three-dimensional constitutive model for the large stretch behavior of rubber elastic materials. J Mech Phys Solids 41(2), 389 - 412.

Bergstrom, J. \& M. Boyce (1998). Constitutive modeling of the large strain time-dependent behavior of elastomers. J Mech Phys Solids 46(5), 931 - 954.

Bérardi, G., M. Jaeger, R. Martin, \& C. Carpentier (1996). Modelling of a thermo-viscoelastic coupling for large deformations through finite element analysis. Int J Heat Mass Transf 39(18), $3911-3924$.

Coleman, B. D. \& W. Noll (1963). The thermodynamics of elastic materials with heat conduction and viscosity. Arch Ration Mech Anal 13(1), 167-178.

Guo, Q., F. Zaïri, \& X. Guo (2018). A thermo-viscoelasticdamage constitutive model for cyclically loaded rubbers. Part I: Model formulation and numerical examples. Int J Plasticity $101,106-124$.

Guo, Q., F. Zaïri, C. Ovalle Rodas, \& X. Guo (2018). Constitutive modeling of the cyclic dissipation in thin and thick rubber specimens. ZAMM - Journal of Applied Mathematics and Mechanics / Zeitschrift für Angewandte Mathematik und Mechanik 98(10), 1878-1899.

Gurtin, M. E. \& L. Anand (2005). The decomposition $\mathbf{F}=\mathbf{F}_{e} \mathbf{F}_{p}$, material symmetry, and plastic irrotationality for solids that are isotropic-viscoplastic or amorphous. Int $J$ Plasticity $21(9), 1686-1719$.

Guth, E. (1945). Theory of filler reinforcement. $J$ Appl Phys 16(1), 20-25.

Lee, E. H. \& D. T. Liu (1967). Finite-strain elastic-plastic theory with application to plane-wave analysis. J Appl Phys 38(1), $19-27$.

Neuhaus, C., A. Lion, M. Johlitz, P. Heuler, M. Barkhoff, \& F. Duisen (2017). Fatigue behaviour of an elastomer under consideration of ageing effects. Int J Fatigue 104, 72 - 80.

Ovalle Rodas, C., F. Zaïri, \& Naït-Abdelaziz (2013). Thermovisco-hyperelastic modeling of the rubber self-heating under fatigue. In Constitutive Models for Rubber VIII, pp. 131-136.

Ovalle Rodas, C., F. Zaïri, \& M. Naït-Abdelaziz (2014). A finite strain thermo-viscoelastic constitutive model to describe the self-heating in elastomeric materials during low-cycle fatigue. J Mech Phys Solids 64, 396-410.

Ovalle Rodas, C., F. Zaïri, M. Naït-Abdelaziz, \& P. Charrier (2016). A thermo-visco-hyperelastic model for the heat build-up during low-cycle fatigue of filled rubbers: Formulation, implementation and experimental verification. Int $J$ Plasticity 79, 217-236. 